\title{
Untersuchungen über Manganverbindungen.
}

Von

Odin T. Christensen.

I.

\section{Über Ammoniumpermanganat.}

Vor einigen Jahren habe ich zur Darstellung verschiedener Manganiverbindungen die Reaktion zwischen Kaliumpermanganat und einem Manganosalz in Gegenwart eines Überschusses der betreffenden Säure empfohlen, ${ }^{1}$ indem die zwei Manganoxyde $\mathrm{Mn}_{2} \mathrm{O}_{7}$ und $\mathrm{MnO}$ dabei nach der Gleichung $\mathrm{Mn}_{2} \mathrm{O}_{7}+8 \mathrm{MnO}=5 \mathrm{Mn}_{2} \mathrm{O}_{3}$ reagierten.

Die Anwendung von Kaliumpermanganat zu dieser Reaktion ist jedoch nicht immer zweckmälsig, denn die dabei erhaltenen Produkte sind oft kaliumhaltig, und wenn man als Manganosalz das Sulfat verwendet, wird auch leicht etwas Schwefelsäure von dem entstandenen Manganisalz zurückgehalten, besonders wenn dieses in Wasser unlöslich ist.

Das erwähnte Prinzip ist indessen zweckmäfsig, namentlich weil. es möglich ist, durch Anwendung der berechneten Mengen von Permanganat und Manganosalz den beabsichtigten Oxydationsgrad des Mangans genau zu erreichen. Es entstand also die Aufgabe, ein Permanganat, dessen Metall, und ein Manganosalz, dessen Säure sich leicht von den gebildeten Produkten entfernen liefsen, zu verwenden. Von vorn herein liefs sich annehmen, dals Ammoniumpermanganat und Manganoacetat mit Vorteil angewandt werden konnten, indem sowohl Ammoniak als Essigsäure wahrscheinlich sich

1 Oversigt over det kgl. danske Vidensk. Selskabs Forhandl. 1896, 94-112. Meine früheren Abhandlungen über Manganverbindungen finden sich im Journ. pr. Chem. (N. F.) (1883) 28, 1; (1886) 34, 41; (1887) 355, 57, 161 und 541. 
leicht aus den Produkten entfernen lassen würden. Manganoacetat läfst sich leicht erhalten. Dagegen mulste ich, um grölsere Mengen von Ammoniumpermanganat zu erhalten, die für die Darstellung dieser Verbindung früher angegebenen Methoden durchprobieren. Bei den Untersuchungen über dieses Permanganat zeigte es sich, dals es mehrere interessante und eigentümliche Eigenschaften besitzt, welche früher nicht hinlänglich beachtet und zum Teil gar nicht bemerkt worden sind.

Dieser Abschnitt meiner Arbeit bespricht ausschliefslich das Ammoniumpermanganat, seine Darstellung und seine Zersetzungsprodukte unter verschiedenen Bedingungen.

\section{Ammoniumpermanganat, $\mathrm{NH}_{41} \mathrm{MnO}_{4}$.}

Mitscherdich ${ }^{1}$ hat dieses Salz durch Behandlung von feingepulvertem Silberpermanganat mit einer Chlorammoniumlösung erhalten. Diese Methode giebt ein reines Produkt, leidet aber an der Unannehmlichkeit, dafs das Chlorsilber, welches bei der Reaktion gebildet wird, sogleich einen grofsen Teil des festen Silberpermanganats einhüllt und dadurch die Einwirkung dieser Verbindung auf die Salmiaklösung bedeutend hemmt, selbst wenn man nach MITSCHERLICH's Vorschrift das Permanganat lange mit dieser Lösung verreibt. Selbstverständlich kann man dieser Unannehmlichkeit entgehen, indem man sowohl das Silberpermanganat als das Chlorammonium in warmem Wasser bei $70^{\circ}$ löst und dann die Lösungen vermischt; da aber das Silbersalz sehr schwerlöslich ist, erfordert es viel Wasser zur Lösung, und man erhält eine ziemlich verdünnte Lösung von Ammoniumpermanganat, was bei der Darstellung gröfserer Mengen dieses Salzes nicht angenehm ist, besonders weil das Salz bei anhaltendem Eindampfen seiner Lösung teilweise zersetzt wird. Dazu kommt die schliefsliche Aufarbeitung des als Nebenprodukt erhaltenen Chlorsilbers. Mit Vorteil habe ich MirscherLich's Methode angewandt, wenn ich kleinere Mengen von reinem Ammoniumpermanganat brauchte.

AschofF ${ }^{2}$ benutzte zur Darstellung von löslichen Permanganaten entweder MitscherLich's Methode oder die Einwirkung von Baryumpermanganat auf das betreffende Sulfat. Das Baryumsalz stellte er aus dem Silbersalz und Chlorbaryum dar, und die Darstellung des Ammoniumsalzes wird dadurch noch weitläutiger.

1 Pogg. Ann. (1832) 25, 296.

* Über die Übermangansäure (Dissert. Göttingen), Berlin 1861, S. 34. 
R. BötTGER ${ }^{1}$ gab eine Methode zur Darstellung von Baryummanganat an und bereitete dann Baryumpermanganat aus diesem Salze durch Einwirkung von Kohlensäure; er empfiehlt zur Darstellung von Ammoniumpermanganat besonders die Doppeltzersetzung zwischen Ammoniumsulfat und Baryumpermanganat - eine Methode, welche vor wenigen Jahren wieder von W. Muthrans ${ }^{2}$ in Vorschlag gebracht worden ist, indem dieser Chemiker gleichzeitig eine leichtere Darstellungsweise für das Baryumsalz mit Kaliumpermanganat als Ausgangsmaterial beschrieb.

Am Schlufs seiner Abhandlung führt Bö'trGGER ${ }^{3}$ mit wenigen Worten an, dafs man auch Ammoniumpermangnat durch Versetzen einer Lösung von Kaliumpermanganat mit überschüssigem Salmiak und Eindampfen der Mischung bis zur gehörigen Krystallisation erhalten kann; dazu bemerkt er, dals man, um das Salz rein zu haben, nur ein einziges Mal umzukrystallisieren nötig hat. -

Es ist einleuchtend dafs die letztgenannte Methode - ihre Richtigkeit vorausgesetzt, - unbedingt vorzuziehen ist, wenn gröfsere Mengen von Ammoniumpermanganat erforderlich sind. Da Börtger indessen nichts näheres über die Ausführung der Methode oder die angewandten Mengenverhältnisse angiebt, habe ich die Methode näher durchprobiert und durchgearbeitet. Dabei hat sich folgendes Verfahren als zweckmälsig erwiesen:

3 Liter Wasser werden im Wasserbade auf $70-80^{\circ}$ erhitzt, dann werden unter Umrühren $160 \mathrm{~g}$ grob gepulvertes Kaliumpermanganat hinzugefügt, und das Erhitzen wird fortgesetzt, bis alles gelöst worden ist. Ist dieses mit Sicherheit konstatiert, dann fügt man unter Umrühren zur heifsen Lösung $440 \mathrm{~g}$ reinen trublierten Salmiak. Dies ist ca. die 8 fache der nach der Gleichung:

$$
\mathrm{KMnO}_{4}+\mathrm{NH}_{4} \mathrm{Cl}=\mathrm{KCl}+\mathrm{NH}_{4} \mathrm{MnO}_{4}
$$

berechneten Menge; ein so bedeutender Überschufs ron Salmiak wird angewandt, um sogleich bei der ersten Krystallisation das Ammoniumpermanganat so wenig kaliumhaltig als möglich zu erhalten.

Das Gemisch wird nun auf dem Wasserbade bei $70^{\circ}$ unter wiederholter Umrührung eingedampft, bis sein Volum ca. 16-1700 ccm beträgt. Dabei tritt einige Zersetzung ein, indem sich eine verhältnismäfsig geringe Menge von höherem Manganoxyd ausscheidet und

1 Journ. pr. Chem. (1863) 90, $156 \mathrm{ff}$.

2 Ber. deutsch. chem. Aes. 1893, 1016.

${ }^{3}$ l. e. 
auf dem Boden der Schale sich absetzt. Diese Zersetzung ist jedoch geringer, wenn das Eindampfen wie beschrieben bei $70^{\circ}$ im Wasserbade stattfindet, als wenn es über offenem Feuer vorgenommen wird; selbstverständlich ist sie bedeutender wenn das angewandte Chlorammonium reduzierende Stoffe enthält. - Wen ndie Lösung auf $16-1700 \mathrm{ccm}$ eingedampft worden ist, lälst man sie einige Minuten ruhig stehen und giefst sie dann von dem ausgeschiedenen höheren Manganoxyd in ein Becherglas über. In diesem Glase bleibt sie his zum nächsten Tage stehon.

Beim Abgiefsen der Mutterlauge wird man dann finden, dafs reichliche Mengen von Ammoniumpermanganat auskrystallisiert sind. Bei der Krystallisation in einem hohen Becherglase erreichen die Krystalle oft Längen von $5-10 \mathrm{~cm}$ und sind aufserordentlich schön. Die ursprüngliche Lösung darf bei der ersten Krystallisation nicht mehr als auf $16-1700 \mathrm{ccm}$ eingedampft werden, denn bei weiterem Eindampfen und Abkiihlen scheidet sich zugleich Salmiak ab. Die Mutterlauge kann durch vorsichtiges Eindampen auf ca. $1500 \mathrm{ccm}^{\prime}$ ein wenig mehr krystallisiertes Permanganat geben, welches jedoch für sich gesammelt werden mufs. - Die gesammelte Ausbeute an rohem Ammoniumpermanganat ist ca. $100 \mathrm{~g}$, der Rest bleibt in der Mutterlauge.

Das Produkt wird sofort durch Umkrystallisation gereinigt. Am zweckmäfsigten trennt man nach Abgiefsen der Mutterlauge die grofsen Krystalle von den kleineren, die sich auf dem Boden des Becherglases ausgeschieden haben und oft mit unlöslichen, höheren Manganoxyden verunreinigt sind. Die grölseren Krystalle werden gesondert umkrystallisiert, indem man zuerst $6-7$ mal soviel Wasser auf dem Wasserbade bis auf $60-70^{\circ}$ erwärmt und dann die Krystalle unter Umrühren hineinbringt; die gebildete Lösung wird bei derselben Temperatur auf ein etwas kleineres Volum eingedampft und dann langsam abgekühlt. Die dabei gebildeten Krystalle werden von der Mutterlauge getrennt, und diese dann weiter bei $60-70^{\circ}$ eingedampft. Die oben erwähnten, bei der ersten Krystallisation erhaltenen kleineren Krystalle werden im Becherglase mit ca. 15 mal soviel Wasser bei gewöhnlicher Temperatur übergossen und man rührt längere Zeit um; dabei werden sie zuletzt gelöst, während die unlöslichen Nanganoxyde zurückbleiben. Un diese zu entfernen, lälst man die Nischung über Nacht stehen und dekantiert dann so sorgfältig als möglich die Lösung von dem Bodensatz, worauf sie 
bei $70^{\circ}$ zur Krystallisation eingedampft wird. Das umkrystallisierte Permanganat wird auf porösem Porzellan im Dunkeln getrocknet.

Von vornherein war es zu erwarten, dafs die oben erwähnte Angabe von Böttger über die Reinheit des Salzes nicht ganz stich-. haltig wäre, da Kalium- und Ammoniumpermanganat isomorph sind. Der Versuch zeigte auch, dafs das zweimal umkrystallisierte Ammoniumpermanganat nicht ganz kaliumfrei war. Für viele Anwendungen ist indessen das Produkt hinlänglich rein; am besten ist es, wenn man das Salz noch reiner zu erhalten wünscht, gröfsere Mengen des Rohprodukts darzustellen und dann das ganze erst aus einer ziemlich starken Salmiaklösung und zuletzt aus Wasser umzukrystallisieren.

Das Mangan wurde in einem zweimal umkrystallisierten Produkt bestimmt: $1.0045 \mathrm{~g}$ lieferten nach Lösen in Wasser, Reduktion mit schwefliger Säure und Salzsäure, Eindampfen der Lösung im Wasserbade zur Trockene, Lösen des Restes in Wasser, Fällen der Lösung mit Ammoniumkarbonat und Glühen des Manganokarbonats $0.5585 \mathrm{~g}$ $\mathrm{Mn}_{3} \mathrm{O}_{4}$ entsprechend $0.4024 \mathrm{~g}$ Mangan oder $40.06 \%$. Berechnet 40.15 .

Wie oben erwähnt ist das Ammoniumpermanganat früher von verschiedenen Forschern untersucht worden; in keinem Falle war jedoch die Untersuchung sehr eingehend, daher sind nur einige seiner Eigenschaften näher bekannt.

Mitscherlich ${ }^{1}$ untersuchte die Krystallform des Salzes, er erwähnt seine Löslichkeit und giebt an, dals die wässerige Lösung des Ammoniumpermanganats ohne Zersetzung eingedampft werden kann, dagegen bemerkt er, dafs Permanganate beim Erhitzen mit Ammoniak Stickstoff geben, indem sowohl Ammoniak als Übermangansäure dabei zerlegt werden. MitscherLich versuchte aus der Menge des entwickelten Stickstoffgases die Zusammensetzung der Übermangansäure zu bestimmen; dieses gelang aber nicht, ,weil sich bei dieser Zersetzung auch eine Verbindung von Stickstoff mit Sauerstoff bildet".

AscноғF ${ }^{2}$ teilt, aufser einer Analyse des Ammoniumpermanganats, mit, dafs dieses Salz sich in 12.6 Teile Wasser löst, und Murhmans ${ }^{3}$ führt zu den früheren Angaben die Beobachtung, dafs die wässerige Lösung des Ammoniumpermanganats beim Kochen unter Stickstoffentwickelung Braunstein ausscheidet, nach der Gleichung

$$
\mathrm{NH}_{4} \mathrm{MnO}_{4}=\mathrm{N}+\mathrm{MnO}_{2}+2 \mathrm{H}_{2} \mathrm{O} \text {. }
$$
1. e.
21. e.
${ }^{3}$ l. c. 
Zugleich beobachtete er, dafs das Salz bei starkem Druck mit einem Pistil in einem Mörser explodierte, indem Ozon und ein aus feinem Braunstein bestehender Rauch entstehen.

Dals das Permanganat sich beim Erhitzen leicht zersetzt, war schon früber bekannt. ${ }^{1}$

Aus den oben angeführten Bemerkungen folgt, dafs das chemische Verhalten des Ammoniumpermanganats nicht eingehend untersucht worden ist. Die Angaben Mitscheruich's und Murumans's über das Verhalten der wässerigen Lösung des Salzes beim Eindampfen und Kochen widersprechen einander zum Teil. Die folgenden Untersuchungen werden zeigen, dafs MuthmanN's Beobachtung über die Zersetzung der Lösung beim Kochen richtig ist, dals sie aber nicht in so einfacher Weise, wie ron ihm angenommen, vorgeht. Der bei der Explosion des Permanganats entstehende "Rauch" ist auch nicht so, wie reiner Braunstein zusammengesetzt.

Die Formel des Ammoniumpermanganats, $\mathrm{NH}_{4} \mathrm{MnO}_{4}$, berechtigt im Voraus zu erwarten, dals die einzelnen Bestandteile des Salzes unter verschiedenen äufseren Bedingungen in verschiedener Weise aufeinander wirken konnten. Die einfachste Reaktion würde die von Muthmans angegebene sein, wobei ein Teil des Sauerstoffs des Moleküls mit dem Wasserstoff Wasser bildet, während der Rest des Moleküls in Mangandioxyd und Stickstoff zerfällt.

Da es indessen bekannt ist, dafs Ammoniak von Übermangansäure zu höheren Stickstoffoxyden oxydiert werden kann, war es nicht unwahrscheinlich, dals eine solche Wirkung unter passenden Bedingungen auch zwischen den Molekülen des Ammoniumpermanganats stattfinden konnte.

Die unten angeführten Versuche werden zeigen, dafs eine solche Reaktion auch in mehreren Fällen eintritt und a. a. zur Salpetersäurebildung Veranlassung giebt, indem als Reaktionsprodukt Ammoniumnitrat entsteht.

\section{Das Verhalten des Ammoniumpermanganats bei der Aufbewahrung.}

Krystallisiertes Ammoniumpermanganat, welches aus dem Kaliumsalz und Salmiak in oben angegebener Weise dargestellt und umkrystallisiert war, wurde unmittelbar nach dem Trocknen in ein gewöhnliches Präparatenglas gebracht, welches dann in verschlossenem Zustande im zerstreuten Taglicht hingestellt wurde.

\footnotetext{
' Berzelius, Lehrbuch (3. Aufl.) 4, 229.
} 
Nach einigen Tagen bemerkte man, wenn der Stöpsel abgenommen wurde, einen schwachen Geruch, ob von Ozon oder Stickoxyd, liel's sich noch nicht sagen; übrigens war kaum eine Änderung in dem Ausschen des Salzes zu beobachten, vielleicht war die Farbe etwas mehr blaugrau. Das Glas wurde wieder verschlossen und blieb darauf wieder in den warmen Sommermonaten 1899 stehen. Es war nun ganz deutlich, dafs das Salz eine langsam fortschreitende Zersetzung erlitt; wenn man in Zwischenräumen von einigen Tagen den Stöpsel des Glases kurze Zeit abnahm, bemerkte man stets einen deutlichen Geruch von Stickstoffoxyden. Nach 3-4 Monaten (Juni-September) waren die Krystalle in solcher Weise zusammengebacken, dal's es unmöglich war, das Permanganat aus dem Glase zu schaffen, es wurde dann mit soviel Wasser in dem Glase übergegossen, dals es eben damit bedeckt war. Das Wasser wurde sogleich violettrot von etwas gelöstem Permanganat, das Glas wurde wieder verschlossen und während $11 / 2--2$ Monate hingestellt, indem in dieser Zeit das Ganze zirka einmal wöchentlich vorsichtig durchgeschüttelt wurde. Am Schlufs dieser Periode verlor die Lösung mehr und mehr ihre Farbe und war zuletzt ganz farblos, selbst wenn sie mit der ungelösten Hauptmenge der Krystalle durchgeschüttelt wurde. Anscheinend waren die Krystalle unverändert, nur ihre Farbe und ihr Glanz war mehr graphitähnlich, und sie waren dem Manganit oder anderen in der Natur vorkommenden krystallisierten höheren Manganoxyden sehr ähnlich. Da sie nicht in Wasser löslich waren, mulste man vorweg annehmen, dafs sie aus höheren Manganoxyden beständen. Eine nähere Untersuchung zeigte, daf́s eine vollständige Umsetzung $z$ wischèn den Bestandteilen des ursprünglichen Ammoniumpermanganats eingetreten war. Eine Probe von der farblosen Lösung im Glase gab beim Kochen mit Natron eine reichliche Ammoniakentwickelung und mit Schwefelsäure und Ferrosulfat eine bedeutende Salpetersäurereaktion.

Das Ammoniumpermanganat war demuach bei der Aufbewahrung in der Sommerwärme unter den erwähnten Bedingungen im Laufe von einigen Monaten vollständig unter Bildung von Ammoniumnitrat zersetzt worden. Das unlösliche krystallinische Produkt bestand im wesentlichen aus höheren Manganoxyden in Pseudomorphosen nach Ammoniumpermanganat (siehe unten). Um alles Ammoniumnitrat zu entfernen, wurden die Krystalle mit kaltem Wasser 
ausgewaschen. Die oben erwähnte farblose Lösung und das Waschwasser wurden vereinigt und lieferten beim Eindampfen im Wasserbade bei $80^{\circ}$ und Erkalten der konzentrierten Lösung krystallisiertes Ammoniumnitrat in verhältnismälsig reichlicher Menge.

Glejchzeitig mit der Zersetzung von Ammoniumpermanganat in höheres Manganoxyd und Ammoniumnitrat treten offenbar auch andere Zersetzungen ein; wie oben angeführt entstehen zugleich Stickstoffoxyde, wahrscheinlich auch etwas freier Stickstoff, und die erwähnten graphitglänzenden Pseudomorphosen, welche nach dem Auswaschen mit Wasser zurückbleiben, enthalten noch Ammoniak. Selbst nach fortgesetztem Auswaschen und Auskochen mit Wasser halten sie hartnäckig etwas Ammoniak zurück.

Die einfachste Bildung von Ammoniumnitrat aus Ammoniumpermanganat würde ohne Nebenreaktionen nach der Gleichung:

$$
2 \mathrm{NH}_{4} \mathrm{MnO}_{4}=\mathrm{NH}_{4} \mathrm{NO}_{3}+2 \mathrm{H}_{2} \mathrm{O}+\mathrm{Mn}_{2} \mathrm{O}_{3}
$$

vor sich gehen.

Nähere Untersuchungen zeigten aber, dafs das zurückgelassene höhere Manganoxyd, (die erwähnten Pseudomorphosen) nicht aus Manganoxyd, $\mathrm{Mn}_{2} \mathrm{O}_{3}$, besteht, sondern der Zusammensetzung eines noch höheren Manganoxyds entspricht, welches zwischen Manganoxyd und Mangandioxyd liegt. Bei der Reaktion wird ein Teil des Ammoniaks zu Stickstoffoxyden oxydiert, welche wahrscheinlich im verschlossenen Glase auf den nicht zersetzten Teil des Permanganats reagiert, aufserdem wird etwas Ammoniak von dem gebildeten höheren Manganhydroxyd gebunden; dieses Hydroxyd ist wahrscheinlich eine schwache Säure und hält daher etwas Ammoniak hartnäckig zurück; selbst wenn die Pseudomorphosen nach Auskochen und Auswaschen mit Wasser 2 Monate an der Luft gelegen hatten, enthielten sie noch ein wenig Ammoniak. Die Zusammensetzung dieser Pseudomorphosen ist unten besprochen.

Den oben erwähnten Versuch, welcher in den Sommermonaten ausgeführt wurde, habe ich später in der Weise wiederholt, dals das krystallisierte Ammoniumpermanganat in einem verschlossenen Präparatglase im Thermostaten bei $25-27^{\circ}$ hingestellt wurde. DerVersuch begann zu Ende des Monats Januar; schon nach einigen Tagen war der Geruch von Stickstoffoxyden merkbar und trat später sehr deutlich hervor. Nach einem Monat war schon ein Teil des Permanganats 
zersetzt, und zu Ende des Monats März war die Spaltung vollständig, obne dafs ich vorher Wasser zugeführt hatte.

\section{Das Verhalten des Ammoniumpermanganats beim Erhitzen.}

Sehon aus früherer Zeit ist es bekannt, dals das Ammoniumpermanganat beim Erhitzen auf höhere Temperatur leicht zersetat wird, doch findet man keine nähere Angabe über die dabei eintretenden Verhältnisse. Erhitzt man wenige Krystalle des Permanganats in einem Platintiegel über der Bunsenlampe, so verpufit das Salz plötzlich und sendet eine feine Staubwolke aus, welche aus höheren Manganoxyden besteht; gleichzeitig bemerkt man einen deutlichen Geruch von Stickstoffoxyden. Das Verpuffungsprodukt ist ein äufserst lockeres und leichtes Pulver, welches wie Rufsflocken niederfällt.

Es war zu erwarten, dals das Ammoniumpermanganat, wenn es schon bei Sommerwärme während einiger Monate der oben bebeschrienen langsamen Zersetzung unterlag, derselben Spaltung in Ammoniumnitrat und höherem Manganoxyd viel schneller bei einer etwas höheyen Temperatur unterliegen würde. Nur mufste diese Temperatur niedriger als die Verpuffungstemperatur liegen. Vorläutige Versuche zeigten, dafs das Permanganat schon nach kurzer Zeit verpufft, wenn es im Trockenschranke auf $80-90^{\circ}$ erhitzt wird, und dafs die Verpuffung schon bei noch niedrigerer Temperatur eintreten kann. Dies geht aus den folgenden Versuchen hervor:

Ca. $2 \mathrm{~g}$ eines Produktes, welches gegen 2 Nonate bei gewöhnlicher Temperatur aufbewahrt worden war und sich daher schon in Zersetzung befand, wurden im Trockenschrank bei $58-60^{\circ}$ hingestellt. Nach mehreren Stunden trat plötzlich Verpuffung ein.

$1.4002 \mathrm{~g}$ von demselben Produkte wurden bei $56^{\circ}$ im Trockenschranke hingestellt: anfangs verlor es ziemlich schnell an Gewicht, später sehr langsam; nach einiger Zeit wurde die Temperatur auf $60^{\circ}$ erhöht. Nach 20 Stunden wog der Rest konstant $0.108 \mathrm{~g}$; der Verlust war demnach $7.7 \%$. An diesem Zeitpunkte hatten die Krystalle ganz ihr ursprüngliches Aussehen bewahrt; ibre Farbe und Glanz war aber mehr graphitännlich oder stahlgrau; sie waren nicht mehr in Wasser löslich, enthielten demnach nicht mehr Amnoniumpermanganat; dagegen liefsen sich im wässerigen Auszug sowohl Salpetersäure als Ammoniak nachweisen. Beim Er- 
hitzen des Permanganats war demnach ebenso wie bei seiner Aufbewahrung Ammoniumnitrat gebildet worden.

Da das zu diesen Versuchen angewandte Ammoniumpermanganat schon in Zersetzung begriffen war, stellte ich nach MiтscherLrCH's Methode aus Silberpermanganat und Salmiak ein reines Präparat dar und wandte sogleich dieses zu folgenden Versuchen an:

Ca. $2 \mathrm{~g}$ von dem reinen Permanganat wurden im Trockenschrank bei $52^{\circ}$ hingestellt und die Temperatur wurde allmählich auf $58^{\circ}$ erhöht; in den ersten 12 Stunden verlor das Salz langsam an Gewicht und sein Aussehen wurde dabei etwas geändert; das Erhitzen wurde noch einige Stunden bei derselben Temperatur fortgesetzt; dann trat plötzlich eine explosionsähnliche Verpuffung ein: es ertönte ein dumpfer Knall und der Geruch von Stickstoffoxyden war sehr deutlich. Das Verpuffungsprodukt war nicht das gewöhnliche, welches am nächsten dem Mangansesquioxyd entspricht, sondern bestand aus zimmetbraunem Mangano-Manganioxyd, aus welchem sich durch Wasser sehr wenig Manganonitrat ausziehen liefs.

Ein neuer Versuch wurde mit demselben reinen Ammoniumpermanganat ausgeführt, indem die Temperatur in 6 Tagen, 8 Stunden täglich, auf $42-46^{\circ}$ gehalten und dann $2-3$ Tage auf $50-54^{\circ}$ und schliefslich einige Stunden auf $60^{\circ}$ erhöht wurde. Dadurch gelang es, der Verpuffung zu entgehen, und das Versuchsresultat entsprach nun ganz dem früher beschriebenen: die Krystalle bewahrten ihre Form ganz unverändert, ihre Furbe wurde dagegen stahlgrau und metallglänzend. Beim vorsichtigen Ausziehen mit Wasser gaben sie Ammoniumnitrat in Lösung und hinterliefsen ammoniakhaltiges höheres Manganoxyd in Pseudomorphosen nach Ammoniumpermanganat.

Damit ist es dargethan, dal's reines Ammoniumpermanganat durch vorsichtiges Erwärmen auf $42-46^{\circ}$ in einigen Tagen und dann auf $50^{\circ}$ dieselbe Zersetzung wie bei der Aufbewahrung während mehrerer Monate bei Sommertemperatur erleidet, indem dadurch Ammoniumnitrat, Stickstoffoxyde und ammoniakhaltiges höheres Manganoxyd gebildet werden.

Das Verhalten des Ammoniumpermanganats beim Kochen mit Wasser.

Wie oben angeführt, hat Mrtschertich angegeben, dals eine wässerige Lösung von Ammoniumpermanganat ohne Zersetzung ein- 
gedampft werden kann, während W. Mutrmans zeigte, dals die Lösung des Salzes beim Kochen unter Bildung von Braunstein und Stickstoff zersetzt wurde.

Es ist oben gezeigt, dals beim vorsichtigen Eindampfen der wässerigen Lösung des Permanganats bei der Darstellung des Salzes immer eine geringe Zersetzung stattfindet, indem etwas höheres Manganoxyd sich dabei ausscheidet. I)as Verhalten des Permanganats beim Erhitzen auf $40-50^{\circ}$ macht es wahrscheinlich, dafs das Salz auch bei fortgesetztem Kochen mit Wasser eine vollständige Zersetzung erleiden würde, obwohl nicht in so einfacher Weise als von Muthmaxy angenommen.

Wenige Gramm reines Ammoniumpermanganat wurden in ca. $750 \mathrm{ccm}$ Wasser gelöst, die Lösung in einen Kolben gebracht und dieser mit aufsteigendem Kühlrohr verbunden; dann wurde der Kolben zuerst auf Drahtnetz 9 Stunden täglich bis zum Kochen der Flüssigkeit erhitzt, nach $2-3$ Tagen trat starkes Stofsen ein, weil sich höhere Manganoxyde ausgeschieden hatten; daher wurde das Erhitzen auf dem Wasserbade fortgesetzt. Nach 8-9 Tagen hatte die Flüssigkeit vollständig ihre Farbe verloren, und ein reichlicher Niederschlag von Manganoxyden war gebildet worden. Nach dem Filtrieren wurde das Filtrat im Wasserbade vorsichtig zur Trockne verdampft; es hinterliefs dabei einen weifsen Rest, welcher sich als Ammoniumnitrat erwies. Der dunkle, beinahe schwarze Niederschlag von höherem Manganoxyd wurde nach dem Auswaschen an der Luft getrocknet, er gab dann noch beim Kochen mit Natron eine deutliche Reaktion auf Ammoniak.

Es ist bei diesem Versuche dargethan, dafs eine verdünnte Ammoniumpermanganatlösung bei fortgesetztem Kochen dieselben Zersetzungsprodukte, Ammoniumnitrat und ammoniakhaltiges Manganoxyd, liefert, die beim vorsichtigen Erhitzen des trockenen Salzes entstehen. Aufserdem entstehen jedoch sicher auch andere Produkte, z. B. Stickstoff, wie von Muthmans angegeben.

\section{Das Verhalten des Ammoniumpermanganats beim Erhitzen mit Ammoniakwasser.}

Es ist bekannt, dals das Kaliumpermanganat beim Erwärmen mit Ammoniakwasser unter Bildung von Stickstoff zersetzt wird. Mit dem Ammoniumsalz ist diese Reaktion noch lebbafter. 
Wird 1 Teil Ammoniumpermanganat in 100 Teilen heifsem Wasser gelöst und dann mit überschüssigem, konzentriertem Ammoniakwasser versetzt, so tritt beim Erhitzen auf dem Wasserbade bald eine lebhafte Stickstoffentwickelung ein, und nach verhältnismäfsig kurzer Zeit ist das Permanganat vollständig zersetzt und die Flüssigkeit farblos, während sich ein Manganoxyd ausgeschieden hat, welches heller braun, als das beim Kochen von dem Permanganat mit Wasser erhaltene Oxyd ist. Die farblose Lösung wurde filtriert und lieferte beim Eindampfen im Wasserbade bei $70-80^{\circ}$ einen kleinen weifsen Rest. Dieser wurde in wenig Wasser gelöst; durch Zusatz von Harnstoff und ein wenig verdünnter Schwefelsäure gab die Lösung beim Erwärmen eine deutliche Kohlensäurereaktion; dies rührt von einem Inhalt des Restes von salpetriger Säure her; nachdem diese durch den Harnstoff zerstört war, gab die Lösung noch eine schwache Salpetersäurereaktion mit Ferrosulfat und Schwefelsäure. Weiter gab der Rest eine starke Reaktion auf A mmoniak.

Ammoniumpermanganat wird demnach beim Erwärmen mit A mmoniakwasserunter Stickstoffentwickelungzersetzt, indem gleichzeitig etwas Ammoniumnitrit, sehr wenig Ammoniumnitrat und aufserdem höhere Manganoxyde entstehen.

Dieses Resultat stimmt mit der Beobachtung von CLOت̈z und GUIGNET u. a. überein, dafs Kaliumpermangat bei gewöhnlicher Temperatur Ammoniak unter Bildung von Kaliumnitrit oxydiert, während bei höheren Temperaturen Kaliumnitrat entsteht.

Das Verhalten des Ammoniumpermanganats gegenüber Salpetersäure. Darstellung von sog. Manganhyperoxydhydrat.

Wie bekannt, giebt Kaliumpermanganat durch Kochen mit Salpetersäure ein Manganhyperoxydhydrat, welches leicht etwas Kalium zurückhält. Zur Darstellung eines kaliumfreien Manganhyperoxydhydrates mulste daher das reine Ammoniumpermanganat besser geeignet sein.

$30 \mathrm{~g}$ Ammoniumpermanganat werden in einen Kolben gebracht und in $400 \mathrm{~cm}$ heifsem Wasser gelöst. Nachdem alles vollständig aufgelöst worden ist, wird der Kolben im Wasserbade erwärmt und zu der $65^{\circ}$ warmen Lösung fügt man dann $50 \mathrm{ccm}$ Salpetersäure vom spezifischen Gewicht 1.4. Die Erwärmung wird fortgesetzt, und wenn die Temperatur der Flüssigkeit $75^{\circ}$ erreicht hat, tritt 
eine ruhige und gleichförmige Gasentwickelung ein. Diese nimmt nach ca. $1 \frac{1}{2}$ Stunde ab und dann werden weitere $25 \mathrm{ccm}$ Salpetersäure hinzugefügt, indem die Erwärmung fortgesetzt wird. $21 / 2$ bis 3 Stunden nach dem Anfang des Versuches ist die Zersetzung vollständig: ein schwarzbrauner Niederschlag von ,Manganhyperoxydhydrat" ist gebildet worden, und die Lösung ist farblos.

Ein in dieser Weise dargestelltes "Manganhyperoxydhydrat"“ wurde zuerst durch Dekantieren und dann auf einer Filterscheibe nit heifsem Wasser und zuletzt mit kaltem Wasser vor der Saugpumpe ausgewaschen, bis es säurefrei war; es wurde an der Luft bei gewöhnlicher Temperatur vollständig getrocknet und bildete dann ein schönes braunschwarzes Pulver. Die Ausbeute war $24 \mathrm{~g}$.

Die Zusammensetzung dieses Hydroxyds wird ersichtlich aus folgender Analyse:

$0.7818 \mathrm{~g}$ von dem vollständig lufttrockenen Produkte verloren bei $100^{\circ}$ $0.1223 \mathrm{~g}$ oder $15.64 \%$ Wasser. Wasser.

$0.6665 \mathrm{~g}$ von demselben Produkt verloren bei $100^{\circ} 0.1043 \mathrm{~g}$ oder $15.64 \%$

$0.7818 \mathrm{~g}$ verloren bei $110^{\circ} 0.1278 \mathrm{~g}$ oder $16.35 \%$ Wasser.

$0.4720 \mathrm{~g}$ branchten nach Behandlung mit Kaliumjodid und Salzsäure $59 \mathrm{ccm}$ Lösung von Natriumthiosulfat, von welchem $1 \mathrm{ccm} 1.1245 \mathrm{~g}$ wirksamen Sanerstoff entsprach; demnach enthalten $0.4720 \mathrm{~g}$ des Hydroxyds $0.06635 \mathrm{~g}$ wirksamen Sauerstoff oder $14.05 \%$.

$0.7685 \mathrm{~g}$ lieferten $0.543 \mathrm{~g} \mathrm{Mn}_{8} \mathrm{O}_{4}$, entsprechend $0.505 \mathrm{~g} \mathrm{MnO}$ oder $65.71 \%$.

Das bei $100^{\circ}$ getrocknete Hydroxyd nimmt an der Luft wieder Wasser auf, anfangs schnell, später langsamer. $0.6665 \mathrm{~g}$, welche bei $100^{\circ} \mathrm{im}$ Gewicht bis auf $0.5622 \mathrm{~g}$ abnahmen, gewannen, der Luft ausgesetzt, wieder Wasser, und wogen dann $0.6225 \mathrm{~g}$ (nicht konstant); wurde das so behandelte Produkt unter einem umgekehrten Becherglase neben ein Glas mit Wasser gestellt, so nahm es weiter an Gewicht zu und wog zuletzt $0.667 \mathrm{~g}$, bekam demnach das ursprüngliche Gewicht. Über Schwefelsäure gestellt, verlor das Produkt wieder $13.05 \%$ Wasser.

Die analytischen Resultate zeigen, dafs das in oben beschriebener Weise aus Ammoniumpermanganat und Salpetersäure erhaltene Manganhyperoxydhydrat eine Zusammensetzung besitzt, welche am nächsten der Formel

entspricht.

$$
22 \mathrm{MnO}_{2} \cdot \mathrm{MnO} \cdot 28 \mathrm{H}_{2} \mathrm{O}
$$

Dieser Formel entspricht ein Gehalt von $65.60 \% \mathrm{MnO}$ und $14.14 \%$ wirksamen Sauerstoff, während $65.71 \% \mathrm{MnO}$ und $14.05 \%$ 
wirksamer Sauerstoff gefunden wurden. $\ddot{\text { Von }}$ den $28 \mathrm{H}_{2} \mathrm{O}$ gehen 22 bei $100^{\circ}$ weg, entsprechend $15.91 \%$ (gefunden $15.64 \%$ ).

Wird das Resultat der Analyse auf wasserfreies Oxyd umgerechnet, dann findet man, dafs das Hydroxyd einem Manganoxyd entspricht, welches

$$
\begin{array}{lll}
\mathrm{MnO} \cdot . \cdot & 82.39 \% \\
\text { wirksames } \mathrm{O} . & 17.61 \%
\end{array}
$$

enthält und die Formel $\mathrm{Mn}_{23} \mathrm{O}_{45}$ besitzt.

Reines wasserfreies Manganhyperoxyd enthält 81.61\% $\mathrm{MnO}$ und $18.39 \%$ wirksamen Sauerstoff.

Das oben beschriebene Manganhyperoxydhydrat hält hartnäckig eine Spur von Ammoniak zurück. Ist das zu seiner Darstellung angewandte Ammoniumpermanganat kaliumhaltig, dann enthält das Hyperoxyd auch eine Spur von Kalium.

Eine vergleichende Untersuchung der in den vorhergehenden Abschnitten dieser Abhandlung erwähnten Manganoxyde, welche als Zersetzungsprodukte des Ammoniumpermanganats unter verschiedenen Bedingungen auftreten, schien von Interesse zu sein; die folgenden Resultate zeigen auch bemerkenswerte Übereinstimmungen.

\section{Untersuchung des durch Aufbewahrung des Ammoniumpermanganats gebildeten höheren Manganhydroxyds.}

Dieses Zersetzungsprodukt besteht, wie oben (S. 209) erwähnt, aus stahlgrauen oder graphitglänzenden Krystallen, Pseudomorphosen nach Ammoniumpermanganat. Es enthält nach dem. Auswaschen und 'Trocknen an der Luft etwas Ammoniak und viel Wasser, und sein Aussehen war dem der natürlich vorkommenden höheren Manganoxyde sehr ähnlich.

Beim Erhitzen des ausgewaschenen und lufttrockenen Produktes entwich Wasserdampf, wenig Ammoniak und Spuren von Stickoxyd.

$0.8750 \mathrm{~g}$ des lufttrockenen Produktes lieferten nach Auflösung in Salzsäure und weiterer Behandlung in gewöhnlicher Weise $0.586 \mathrm{~g} \mathrm{Mn}_{3} \mathrm{O}_{4}$, entsprechend $0.545 \mathrm{~g} \mathrm{MnO}$ oder $62.28^{\circ} \%$.

$0.6275 \mathrm{~g}$ brauchten nach Behandlung mit Jodkalium und Salzsäure $103.8 \mathrm{cml} \% / 10^{-}$-10rm. Natriumthiosulfat $(1 \mathrm{ccm} \sim 0.0008 \mathrm{~g} \mathrm{O})$, entsprechend $0.08304 \mathrm{~g}$ wirksamer Sauerstoff oder $13.23 \%$.

$0.9080 \mathrm{~g}$ verloren bei $95-100^{\circ} 0.1435 \mathrm{~g}$ oder $15.80 \%$.

Das auf $100^{\circ}$ bis zum konstanten Gewicht erhitzte Produkt gab noch eine schwache Ammoniakreaktion mit Natron und reichlich Wasser beim Erhitzen auf höhere Temperaturen. 
Berechnet man nach dem vorliegenden Analyseresultat die $\mathrm{Zu}$ sammensetzung des dem Hydroxyd entsprechenden Manganoxyds, dann findet man, dafs $62.28+13.23 \mathrm{~d}$. h. 75.51 Th. ron diesem Oxyd 62.28 $\mathrm{MnO}$ und 13.23 wirksamen Sauerstoff enthaiten. Auf 100 Teile berechnet wird die Zusammensetzung des Oxyds:

$$
\begin{aligned}
& \mathrm{MnO} \text {. . . } 82.48 \% \\
& \text { wirksamer } 0 \text {. } 17.52 \text {, }
\end{aligned}
$$

Vergleicht man dieses Resultat mit dem oben für das Zersetzungsprodukt des Ammoniumpermanganats durch Kochen mit Salpetersäure gefundenen, dann sieht man, dafs beide vollständig übereinstimmen. Die Pseudomorphosen und das „Manganhyperoxydhydrat" aus Ammoniumpermanganat und Salpetersäure entsprechen demselben höheren Manganoxyd $\mathrm{Mn}_{23} \mathrm{O}_{45}$.

\section{Höheres Manganoxyd, durch anhaltendes Kochen einer wässerigen Ammoniumpermanganatlösung erhalten.}

Das S. 213 erwähnte Manganhyperoxydhydrat, welches durch achttägiges Kochen einer verdünnten Ammoniumpermanganatlösung entstand, bildete nach dem Auswaschen und Trocknen an der Luft ein beinahe schwarzes Pulver, welches beim Erbitzen viel Wasser abgab.

$0.4802 \mathrm{~g}$ von dem vollständig lufttrockenem Produkt verloren bei $100^{\circ}$ $0.0947 \mathrm{~g}$ oder $19.72 \%$ und lieferten $0.3162 \mathrm{~g} \mathrm{Mn}_{3} \mathrm{O}_{4}$, entsprechend $0.2945 \mathrm{~g}$ $\mathrm{MnO}$ oder $61.32 \%$.

$0.511 \mathrm{~g}$ brauchten nach Behandlung mit Jodkalium und Salzsäure $83.6 \mathrm{ccm}$ $2 / 10$-norm. Natriumthiosulfat, entsprechend $0.06688 \mathrm{~g}$ wirksamer 0 oder $13.09 \%$.

Berechnet man ganz wie oben die Zusammensetzung des dem Hyperoxydhydrat entsprechenden höheren Manganoxyds, so findet man, dafs dieses in 74.41 Teilen $61.32 \mathrm{MnO}$ und $13.09 \%$ wirksamen Sauerstoff enthält, oder in Prozenten:

$$
\begin{aligned}
& \mathrm{MnO} \text {. . . } 82.40 \text {, } \\
& \text { wirksamer } O \text {. } 17.60 \text {. }
\end{aligned}
$$

Demnach findet sich das Mangan in diesem Zersetzungsprodukt in demselben Oxydationsgrad wie in den zwei vorhergehenden.

Das Resultat der Untersuchungen äber die bei den Zersetrungen von Ammoniumpermanganat gebildeten höheren Manganhydroxyden ist demnach, dals diese Hydroxyde, wenn sie unter den oben geschilderten Verbältnissen ge- 
bildet sind, alle das Mangan in demselben Oxydationsgrad enthalten, entsprechend dem Oxyd $22 \mathrm{MnO}_{2} \cdot \mathrm{MnO}$ oder $\mathrm{Mn}_{23} \mathrm{O}_{45}$, sei es, dafs das Hydroxyd durch freiwillige Zersetzung des Permanganats bei Sommerwärme oder durch Kochen des Permanganats mit Salpetersäure oder durch anhaltendes Kochen einer verdünnten wässerigen Ammoniumpermanganatlösung zur vollständigen Dekomposition gebildet wäre. Die Wassermenge in den Hydroxyden kann dagegen etwas rerschieden sein.

Höheres Hanganozyd, durch Erwärmen von Ammoniumpermanganatlösung mit Ammoniakwasser orhalten.

Wie sich erwarten liefs, entspricht das bei diesem Prozesse gebildete Hydroxyd einer etwas niedrigeren Oxydationsstufe des Mangans, indem das Ammoniak reduzierend wirkt. Es ist auch oben erwähnt (S. 214), dals die Farbe des Zersetzungsproduktes heller war als die der vorgenannten Hydroxyde. Das Produkt wurde nach Auswaschen erst an der Luft und dann bei $90-100^{\circ}$ getrocknet; dann lag es einige Tage der Luft ausgesetzt, wobei es wieder Wasser aufnahm.

$0.6865 \mathrm{~g}$ von dem in dieser Weise behandelten Hydroxyd lieferten $0.5425 \mathrm{~g} \mathrm{Mn}_{3} \mathrm{O}_{4}$, entsprechend $0.50456 \mathrm{~g} \mathrm{MnO}$ oder $73.49 \%$.

$0.3585 \mathrm{~g}$ brauchten nach Behandlung mit Jodkalium und Salzsäure $56.7 \mathrm{ccm}$ $2 / 10$-norm. Natriurnthiosulfat, entsprechend $0.04536 \mathrm{~g}$ wirksamer $\mathrm{O}$ oder $12.65 \%$.

Demnach entspricht das Hydroxyd einem Manganoxyd von der Zusammensetzung

$$
\begin{aligned}
& \mathrm{MnO} \cdot . \cdot \cdot 85.31 \% \\
& \text { wirksamer O } \\
& \cdot 14.69 \%
\end{aligned}
$$

Die oben beschriebenen Untersuchungen zeigen, dafs die Beständigkeit des Ammoniumpermanganats verlıältnismärsig gering ist; seines losen Baues wegeı ist es aber für mehrere Reaktionen anwendbar, nur muls man es kurze Zeit vor seiner Anwendung darstellen oder jedenfalls in einem küblen Raum aufbewahren, denn die Zersetzung. welche sich in Sonnenwärme in einigen Monaten vollendet, tritt auch, obwohl bedeutend langsamen, bei $15^{\circ}$ ein und ist durch den schwachen Geruch von Stickoxyd merkbar.

Die explosiven Figenschaften des Ammoniumpermanganats lassen 
sich am besten in der Weise demonstrieren, dafs man zuerst ein paar Krystalle auf einem Ambofs oder einer anderen Eisenunterlage leise zerdrückt, und dann mit einem Hammer einen kräftigen Schlag darauf richtet. És ertönt dann ein scharfer Knall, und man bemerkt, wie MUтнмаN angegeben hat, einen deutlichen Geruch von Ozon.

Die Anwendungen des Aminoniumpermanganats zur Darstellung von anderen Manganverbindungen werde ich in den folgenden Abhandlungen gelegentlich erwăhnen. Dic zunächst folgende Abhandlung beschreibt insbesondere die Bedingungen der Bildung von Manganalaunen.

Kopenhagen, Chem. Laboratorium der kgl. landw. Hochschule, April 1900. Bei der Redaktion eingegsngen am 27. April 1900. 